\title{
Muscle strength does not explain standing ability in children with bilateral spastic cerebral palsy: a cross sectional descriptive study
}

\author{
Cecilia Lidbeck*, Kristina Tedroff and Åsa Bartonek
}

\begin{abstract}
Background: In bilateral cerebral palsy (CP) muscle strength is considered important for development of gross motor functions, but its influence on standing ability has not been explored. Our aims were to examine muscle strength with respect to the ability to stand with (SwS) or without (SwoS) hand support, asymmetrical weight bearing (WB), and whether the ability to produce strength was influenced by different seated conditions.

Methods: In this cross sectional descriptive study standing posture was recorded with 3D motion analysis, and muscle strength was measured with a hand-held dynamometer, in 25 children with bilateral CP, GMFCS levels II-III, SwS ( $n=14$, median age 11.4 years), or SwoS, ( $n=11$, median age 11.4 years). Strength measurements were taken in the hip flexors, knee extensors, dorsiflexors and plantarflexors, in two seated conditions; a chair with arm- and backrests, and a stool.

Results: Compared to SwoS, children SwS stood with a more flexed posture, but presented with equal strength in the hip flexors, dorsiflexors and plantarflexors, and with somewhat more strength in the knee extensors. Despite asymmetric WB during standing, both limbs were equally strong in the two groups. No differences in strength were measured between the two seated conditions.

Conclusions: Despite challenges measuring muscle strength in $\mathrm{CP}$, the lower limb muscle strength cannot be considered an explanatory factor for variations in standing in this group of children with bilateral CP. The findings rather strengthen our hypothesis that deficits in the sensory systems could be as determinant for standing as muscle weakness in children with bilateral spastic CP.
\end{abstract}

Keywords: Asymmetry, Cerebral palsy, Child, Isometric muscle strength, Posture

\section{Background}

Cerebral palsy (CP) is the most common childhood motor impairment in developed countries and describes a group of permanent disorders of the development of movement and posture. The motor disorders are often accompanied by disturbances of sensation, perception and cognition [1]. In bilateral $\mathrm{CP}$ muscle strength is considered important for development of gross motor functions, but its influence on standing ability has not been explored. Muscle weakness is recognized as a primary symptom in children with $\mathrm{CP}$ and children with spastic $\mathrm{CP}$ are substantially

\footnotetext{
* Correspondence: cecilia.lidbeck@ki.se

Department of Women's and Children's Health, Karolinska Institutet, Stockholm, Sweden
}

weaker compared to typically developing (TD) children in the lower limb muscle groups, most prominently in the ankle and the hip muscles $[2,3]$. When muscle strength in $\mathrm{CP}$ was analyzed from a gross motor function classificaotor function, GMFCS level I, proved to be the tor both gross motor function development and tanding and some may require hand-support. It is dependent on muscle strength or if there are other factors affecting the ability to attain standing in $\mathrm{CP}$. 
Muscle strength is clearly important for achieving gross motor functions. Since children with CP are weak, resistance training to achieve functional benefits has become a commonly recommended intervention. Disappointingly, functional gains following resistance training in children with $\mathrm{CP}$ have been hard to demonstrate even though increased muscle strength was detected $[7,8]$. In children with CP the motor disorders are often accompanied by disturbances of sensation and perception [1]. Investigation on how deficits in the sensory systems affect the motor disorder in CP are reported in few studies only. The discrepancy between the children's gross motor function score and how they use their motor function in everyday life, has been hypothesized to be associated with perceptual disorders [9]. In another study by Ferrari et al., an impaired motor control strategy with ineffective anticipatory postural adjustments during a seated reaching task in children with bilateral $\mathrm{CP}$, was explained by disturbances in the perceptual system [10]. Difficulties in producing antigravity reactions during standing have been proposed to relate to perceptual problems [11]. Furthermore, Damiano et al. found that deficits in proprioception with impaired detection of joint position in the lower limbs were linked to postural instability in standing in children with mild bilateral CP [12]. The above mentioned findings indicate that disorders in the sensory and perceptual systems affect gross motor function in $\mathrm{CP}$ even if they are not fully understood.

Compared to children with typical development (TD), children with bilateral CP often present with a flexed standing posture. We have previously found that children stood with more flexion than their potential passive joint angle, showing that they had less restricted knee extension in a non-weight-bearing supine position than in a weight bearing (WB) standing condition [13]. According to the 2007 $\mathrm{CP}$ definition, some children categorized with primarily bilateral involvement may have considerable asymmetry across sides [1]. In our previous study, the assessment of standing posture in children with bilateral $\mathrm{CP}$ revealed a more apparent asymmetrical alignment in the children who stood independently compared to those who needed support [13]. When investigating muscle strength between the more and the less involved limb results indicated that there were no differences in muscle strength between the limbs in bilateral CP [3].

When measuring isometric muscle strength, information about the ability to produce force at a specific, positiondependent muscle length is provided. Measuring muscle strength in children, and in particular in children with brain lesions has been reported to be demanding and deserves careful standardization of measuring positions and data comparisons [14-16]. The reliability of measuring muscle strength in children with $\mathrm{CP}$ is challenging due to poor selective motor control, co-contraction, and the restriction of joint range of motion [3, 14, 17]. Still, measuring muscle strength with a hand-held dynamometer (HHD) in children with $\mathrm{CP}$ has been reported as reliable in the lowerextremities and is a commonly used method in clinical practice $[15,18,19]$. Although, the value of measuring muscle strength in one position and then relate results to gross motor functions, where the muscles are working in other lengths, have been questioned [14].

To our knowledge, there is no information about the role of muscle strength for standing ability in children with CP. The aims were therefore: (i) to explore the lower limb muscle strength in children with various standing abilities, both those requiring assistive device and those who stood unsupported; (ii) to investigate muscle strength with respect to asymmetric WB during standing; and (iii) whether children's ability to produce strength was influenced by different seated conditions. Based on the theory that in CP, the motor disorders are accompanied by disturbances of sensation and perception [9-12], our hypothesis was that in children with bilateral $\mathrm{CP}$, sensory disorders would be as determinant as muscle weakness for standing function in $\mathrm{CP}$.

\section{Methods \\ Participants}

In this cross sectional descriptive study standing posture and muscle strength were investigated in children and adolescents with CP. Participants were consecutively recruited through the neuropaediatric department of Karolinska University Hospital in Stockholm, Sweden, between January 2012 and September 2013. The inclusion criteria were; bilateral CP, 7-17 years of age, GMFCS level II-III, the ability to maintain standing with $(\mathrm{SwS})$ or without $(\mathrm{SwoS})$ hand support for at least $30 \mathrm{~s}$, and the ability to follow verbal instructions. Exclusion criteria were; presence of dystonia, botulinum toxin injections or soft tissue surgery within the past six months, or skeletal surgery within the past year. Informed consent was obtained, verbally from the children and written from the parents. Approval was obtained by the Regional Ethical Review Board in Stockholm, Sweden.

\section{Physical examination}

All children underwent a physical examination that included measurements of joint range of motion (ROM) [20]. If passive ROM was less than the neutral position of a joint, the joint was considered to have a contracture. Spasticity in the lower limbs was also assessed and was documented as either present or absent (Table 1) [21].

\section{Motion analysis}

The standing posture was recorded with a three-dimensional (3D), eight camera motion analysis system (Vicon MX40, Oxford, UK) using a full-body biomechanical model (Plug-In-Gait, Vicon $^{\circ}$ ) with retro-reflective markers, during $30 \mathrm{~s}$ while standing on two force plates (Kistler, Winterthur, Switzerland). The children who needed hand support to 
Table 1 Characteristics of children with $C P$, standing with (SwS) and without (SwoS) hand support

\begin{tabular}{|c|c|c|}
\hline & SwoS $(n=11)$ & SWS $(n=14)$ \\
\hline Age, median (range), years & $11.4(7.7-15.9)$ & $11.4(7.7-17.2)$ \\
\hline Weight, median (range), kg & $45.0(30.7-99.7)$ & $37.3(18.5-65.2)$ \\
\hline Height, median (range), cm & $150.0(124.0-166.0)$ & $141.0(110.0-170.0)$ \\
\hline Female/male & $7 / 7$ & $5 / 6$ \\
\hline GMFCS Level & $\|: 9\| l: 2$, & ॥: 1, II: 13 \\
\hline \multicolumn{3}{|c|}{ Joint contractures, uni or bilateral ${ }^{a}$} \\
\hline Hip flexion contracture & 4 & 8 \\
\hline Knee flexion contracture & 4 & 11 \\
\hline Plantarflexion contracture & 5 & 5 \\
\hline \multicolumn{3}{|l|}{ Spasticity, uni or bilateral ${ }^{a}$} \\
\hline Hip flexors & 1 & $2 / 13$ \\
\hline Knee flexors & $10 / 10$ & 14 \\
\hline Plantarflexors & 11 & 14 \\
\hline \multicolumn{3}{|l|}{ Orthopaedic surgery ${ }^{a}$} \\
\hline Femoral osteotomy & 3 & 3 \\
\hline Adductor tenotomy & 0 & 1 \\
\hline Hamstrings lengthening & 0 & 1 \\
\hline $\begin{array}{l}\text { Calf (Strayer, Achilles } \\
\text { tenotomy) }\end{array}$ & 6 & 4 \\
\hline
\end{tabular}

achieve and maintain standing stood in front of a height-adjustable frame and held the handrail with a slightly flexed elbow position. All children were tested barefoot. The more WB limbs were determined from force plate data. The mean and standard deviation (SD) sagittal plane trunk and pelvis segment, and hip, knee and ankle joints angles from each child's standing trial were used to describe standing posture.

\section{Muscle strength measurements}

A hand-held dynamometer (Chatillon ${ }^{\circ}$, Greensboro, NC, USA) was used to quantify isometric muscle strength. Four muscle groups were tested bilaterally: hip flexors, knee extensors, ankle dorsiflexors, and ankle plantarflexors. Lever arms were measured from the greater trochanter, lateral knee joint, and lateral malleolus to the dynamometer's perpendicular placement. The strength measurements were carried out in randomized order in two seated conditions; a chair with arm- and backrests (chair), and a stool after removing arm- and backrests (stool).

Children were instructed to place their hands on their laps during measurements, and not to lean against the armrest or the backrest. The hip flexors and knee extensors were tested in $90^{\circ}$ hip flexion and $90^{\circ}$ knee flexion as previously described by Eek et al. (Table 2) [22]. To avoid possible restrictions from tight hamstring and/or gastrocnemius muscles the dorsiflexors and plantarflexors were
Table 2 Test positions for isometric muscle strength measurements with hand-held dynamometer

\begin{tabular}{llll}
\hline & Muscle & Position in sitting & Lever arm from \\
\hline & Hip flexors & Hip and knee & Greater trochanter \\
flexed $90^{\circ}$ & \\
\hline & &
\end{tabular}
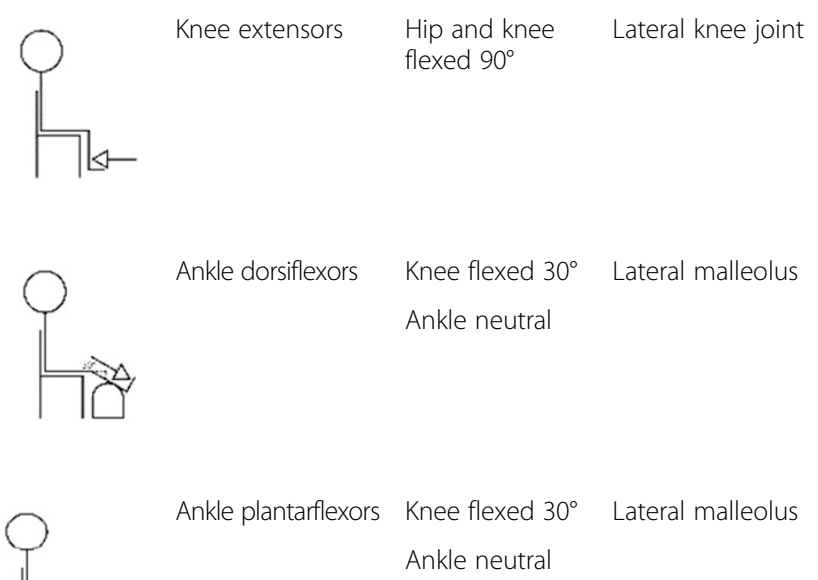

tested in $90^{\circ}$ hip flexion and $30^{\circ}$ knee flexion. The ankle was passively placed in a neutral position. If the range of motion in the ankle was limited, the HHD was placed as close to neutral position as possible [3].

The "make test technique" [14] was used by verbally encouraging the children to press as hard as possible against the dynamometer to build up strength for $4-5 \mathrm{~s}$. Between the trials there was a break of approximately $20 \mathrm{~s}$. To ensure understanding children first completed a familiarization trial. Three trials for each muscle group were conducted in each seated condition. The same examiner (CL) performed all measurements while another controlled for standardization of the testing positions. The force value derived from the dynamometer was multiplied with the lever arm to express strength as torque, which was in turn normalized to body weight. Muscle strength data was analyzed with respect to children's standing ability, more-WB and less-WB limb and the seated conditions.

\section{Statistical analysis}

Non-parametric statistical analyses were carried out using the commercially available software SPSS v.21 Chicago, IL, USA, at a significance level of $\mathrm{p} \leq 0.05$. A Mann-Whitney $U$ test was used for group comparisons between SwS and SwoS. A Wilcoxon's Signed- Rank Test was used to compare muscle strength between the 


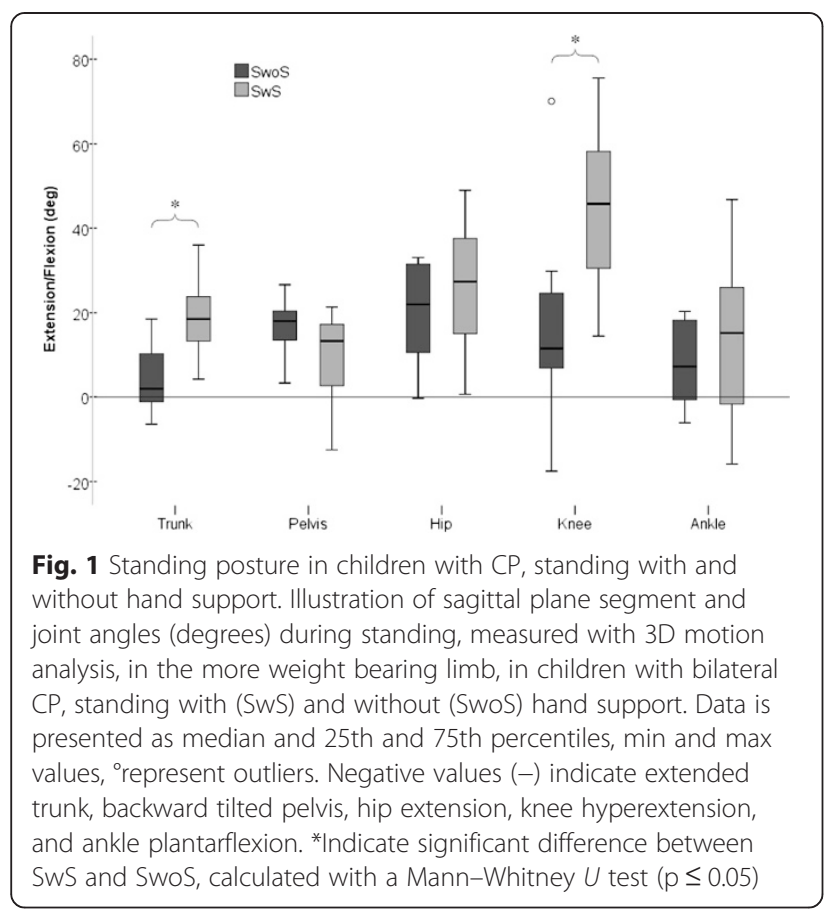

more-WB and less-WB limbs and chair or stool conditions. A Chi-Square test was used to compare incidence of joint contractures, spasticity, and performed orthopedic surgery in the lower limbs between the two groups.

\section{Results}

\section{Participants}

Of the 27 children who were enrolled in the study, two did not complete the examinations; thus 25 children (12 females and 13 males), median age 11.4, (range 7.7-17.2) years participated. According to standing ability 14 children required hand support during standing $(\mathrm{SwS})$, and 11 were able to stand without hand support (SwoS) (Table 1).

No differences were found between children in the SwS-group or SwoS-group in age, weight, height, gender, presence of spasticity or amount of orthopedic surgery. Knee flexion contractures were significantly more frequent in children SwS vs SwoS $(p=0.049)$. The number of contractures were equally distributed in the hip $(p=0.428)$ and the ankle $(p=0.697)$ (Table 1). Among the children with contractures, in SwS and SwoS respectively, the passive ROM median (range) in hip extension was $-5^{\circ}\left(-5-(-15)^{\circ}\right.$ and $-12.5^{\circ}\left(-10-(-20)^{\circ}\right.$, in knee extension: $-15^{\circ}\left(-10-(-25)^{\circ}\right.$ and $-15\left(-5-(-25)^{\circ}\right.$, and in the ankle dorsiflexion: $-5^{\circ}(-5-$ $(-20)^{\circ}$ and $-5^{\circ}\left(-5-(-25)^{\circ}\right.$. Minus is indicating presence of a flexion contracture.

\section{Standing posture}

On a group level, the 3-D motion analysis showed that all children stood with forward leaning trunks, anteriorly tilted pelvis, flexed hips and knees, and with the ankle in dorsiflexion. There was large heterogeneity within the SwoS and SwS groups. Compared to children in SwoS, the children in SwS stood with significantly more forward trunk lean, 19.2 versus $4.4^{\circ}(\mathrm{p}<0.001)$, and with more knee flexion, 44.4 versus $15.8^{\circ}(p=0.001)$. The joint angles in SwS were also somewhat greater in the hip and ankle, though not significantly (Fig. 1).

Differences in weight distribution between the limbs were identified in all children. The more-WB limb was generally more extended in both SwS and SwoS, although there were large intragroup variations. For both children SwoS and SwS the hip was significantly less flexed in the more-WB limb, $19.9^{\circ}$ vs $24.8^{\circ}(p=0.026)$ and $25.9^{\circ}$ vs $34.4^{\circ}(p=0.006)$ respectively. In addition the SwS group had a more extended knee in the moreWB limb, $44.4^{\circ}$ vs $52.6^{\circ}(p=0.041)$. No differences were observed in the ankle.

\section{Muscle strength}

Seventeen of the 25 children included in the study lost attention during testing and completed one trial only in each muscle group in the second position. The first trial from each muscle group was therefore, used for analysis.

There were no differences in muscle strength between the limbs with respect to asymmetrical WB in the hip flexors, knee extensors, or the plantarflexors in either the SwS-group or the SwoS-group. Only the dorsiflexors showed significantly higher strength in the more-WB limb in children SwS (Table 3).

Between the two groups in the more-WB limb, the knee extensor strength values were significantly higher in SwS than in SwoS $(p=0.038)$. No significant differences between SwS and SwoS were found in the hip flexors $(p=0.767)$,

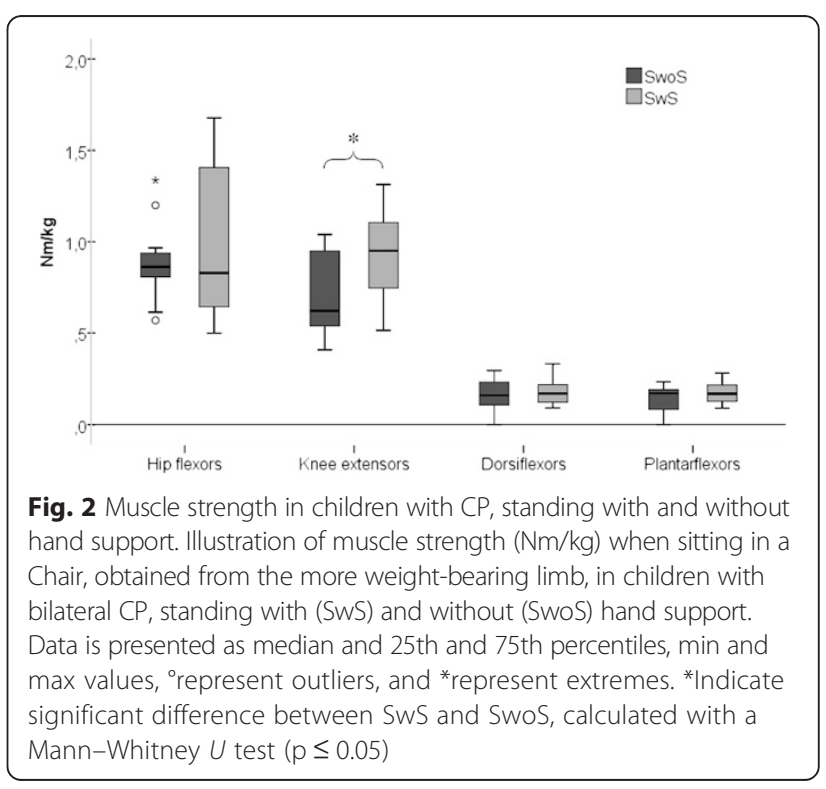


Table 3 Muscle strength in children with CP standing with (SwS) and without (SwoS) hand support

\begin{tabular}{|c|c|c|c|c|c|}
\hline & \multicolumn{2}{|l|}{ More WB-limb } & \multicolumn{3}{|l|}{ Less WB-limb } \\
\hline & Median (Range) Nm/kg & $n$ & Median (Range) Nm/kg & $\mathrm{n}$ & P \\
\hline \multicolumn{6}{|l|}{ SwoS $(n=11)$} \\
\hline Hip flexors & $0.86(0.57-1.34)$ & 11 & $0.81(0.52-1.37)$ & 11 & 0.790 \\
\hline Knee extensors & $0.62(0.41-1.04)$ & 11 & $0.82(0.39-1.24)$ & 11 & 0.328 \\
\hline Dorsiflexors & $0.16(0.00-0.30)$ & 10 & $0.15(0.00-0.30)$ & 11 & 0.441 \\
\hline Plantarflexors & $0.17(0.00-0.23)$ & 9 & $0.15(0.00-0.33)$ & 9 & 0.575 \\
\hline \multicolumn{6}{|l|}{ SWS $(n=14)$} \\
\hline Hip flexors & $0.83(0.50-1.68)$ & 14 & $1.04(0.53-1.79)$ & 14 & 0.331 \\
\hline Knee extensors & $0.95(0.51-1.31)$ & 14 & $0.87(0.44-1.56)$ & 14 & 0.177 \\
\hline Dorsiflexors & $0.17(0.09-0.33)$ & 13 & $0.12(0.08-0.24)$ & 13 & 0.034 \\
\hline Plantarflexors & $0.17(0.09-0.28)$ & 13 & $0.17(0.08-0.27)$ & 13 & 1.000 \\
\hline
\end{tabular}

P-values refer to comparisons between the limbs, significant difference is bolded $(p \leq 0.05)$

$n=\mathrm{nr}$ of children

dorsiflexors $(p=0.976)$, or the plantarflexors $(p=0.431)$ (Fig. 2).

There was no statistical difference in muscle strength between the seated conditions in either SwS or SwoS.

\section{Discussion}

Measuring isometric muscle strength in children with $\mathrm{CP}$ is associated with uncertainties not least because of their deficits in performing voluntary movements [23]. Despite difficulties involved with the measurements, strength does not seem to be a determining factor in whether the children with $\mathrm{CP}$ need support during standing; only minor differences were found in lower limb muscle strength between children standing with or without support. The need for support, as well as a more apparent crouched standing posture in the same individuals, might lead us to believe that difficulties during standing originate from muscle weakness. But contrary to this assumption, the children in this study, were equally strong in their hip and ankle muscles, and the children who required support were somewhat stronger in the knee extensors. The strong knee muscles, however, might be a consequence of the more flexed knees during standing observed in the children SwS. On the other hand, these children stood not only with increased knee flexion, but with a more forward leaning trunk compared to the children in SwoS. As an effect of the flexed body position, the projection of the ground reaction force can be assumed as shifted anteriorly, reducing the internal knee extension joint moment and therefore decreasing the required effort of the knee muscles. Accordingly, the crouched posture during standing in this group of children may not have required much stronger knee muscles than in those standing independently. Furthermore, calf muscle weakness causing instability in the ankle joint may contribute to the flexed knee position during standing. In our study group, the plantarflexors were equally strong in both groups and there were no differences in the amount of calf muscle surgery between the groups. Secondary musculoskeletal problems with decreased joint ROM is frequently observed in children with CP $[1,24]$. In our study, hip, knee and ankle contractures were equally pronounced in both groups, whereas knee flexion contractures were more common in the SwS-group. The knee contractures were not believed to negatively influence the children's ability to produce maximal knee extensor force, as the measurements were performed with a $90^{\circ}$ flexed knee.

In a previous study we reported that children with need for support in standing, stood with less knee extension than was passively available in a non-weight bearing position [13]. This finding could be confirmed in the present study where the children stood with more flexed knees than necessary with respect to their passive ROMs. Not utilizing the full possible knee extension range may indicate difficulties in producing antigravity reactions during standing as a consequence of perceptual problems [11]. Furthermore, impaired proprioception in the lower limbs has been reported to be associated with postural instability in bilateral $\mathrm{CP}$ and could have contributed to children's difficulties to extend their legs against gravity [12].

In bilateral $\mathrm{CP}$, an uneven weight bearing is frequently observed during standing, giving an impression of asymmetric muscle strength. We found that the children's more-WB limb was more extended both in the children in SwS or SwoS. We hypothesized that the support limb, i.e. the more WB limb was the strongest, but this could not be verified despite an asymmetrical loading on the limbs while standing. This is in accordance with Wiley et al. who found both limbs equally strong in children with bilateral CP [3]. 
Even though there are known uncertainties regarding strength measurements in $\mathrm{CP}$, the strength values obtained in this study correspond to previous findings [25]. Dallmeijer et al. employed a method similar to ours and showed equivalent muscle strength values in the knee extensors, dorsiflexors, and plantarflexors in 25 adolescents with bilateral CP, GMFCS level II-III [25]. In accordance with earlier studies comparing muscle strength between GMFCS levels II and III, the children in our groups, SwS and SwoS, were equally strong in the hip flexors and knee extensors when measured in $90^{\circ}$ knee flexion $[2,5]$. However, when measured in $30^{\circ}$ knee flexion, Thompson et al. reported children in GMFCS level III to be weaker in the knee extensors compared with children in level II [5]. This may be explained by the more severe motor disorder in GMFCS level III with difficulties in performing voluntary movements with an almost extended knee. Contrary to the findings of Eek et al. who reported stronger dorsiflexors and plantarflexors in children in GMFCS level II compared to III [2], we found these muscle groups to be equally strong in both groups. The testing positions used in our study were chosen in order to, to the best of our knowledge, reduce the impact of spasticity, tight muscles, and co-contraction [14]. For example the testing of the dorsiflexors in a sitting position, with a slightly flexed knee, avoiding constraint from tight muscles could have enhanced the children's ability to produce a maximal voluntary contraction. The possibility to observe the foot during testing may also have supported the ability to selectively perform the dorsiflexion as well as to compensate for probable difficulties in proprioception $[14,26]$. On the other hand in our study, there were three children who could not undertake strength measurements of the calf muscles due to poor selective motor control distally, in the plantarflexors. All three had a passive range of motion in the ankle to at least neutral position, hence it was not reduced mobility in the ankle that prevented children to perform the movement. Worth noting is that two of these children had the ability to stand without support and therefore, could be expected to have a milder motor disorder compared to children who required support for standing.

To examine whether the children's ability to produce strength was influenced by various seated conditions, the measurements were conducted in a stable sitting position on a chair and in a more demanding sitting position on a stool. Contrary to Ferrari et al. we could not find any differences between the two conditions and speculate that children in our study were allowed to remain within their safe base of support at both measurements. In the above mentioned study, children's limits of stability were provoked during a seated reaching task, thus our method might not have been challenging enough for the sensory-motor system to elicit possible perceptual impairments $[9,10]$.
A limitation of our study was the choice to use the first trial from each seated condition for the analyses. In order to compare muscle strength between groups, it has been recommended to use the highest value alternative an average of two trials for statistical analyses [27, 28]. However, most of the children had trouble to maintain attention during the testing, and it was demanding to accomplish three strength measurements per muscle group in the two seated conditions. Calculations on the maximum strength values from each muscle group were carried out with the same outcome and therefore, the choice to use data from the first trial does not seem to have affected the results. When using an HHD it is difficult to separate out forces caused by strength from spasticity or non-neural components as contractures. Since spasticity in the calf muscle could be expected to be easily elicited during the measurements as well as some children had contractures in the ankle, plantar flexor data from our study must be interpreted with caution. Our results though, were remarkably similar to the values from the Australian study, conducted on adolescents with CP [25].

\section{Conclusions}

Despite the above discussed limitations and those associated with muscle strength measurement in children with $\mathrm{CP}$ in general, our results indicate that the children who required hand support compared to those who stood independently were not weaker in the lower limb muscles. Thus, lower limb muscle strength cannot be considered an explanatory factor for variations in standing in this group of children with bilateral $\mathrm{CP}$. The findings rather strengthen our hypothesis that deficits in the sensory systems, such as proprioception and perception of gravity, could be as determinant for standing as muscle weakness in children with bilateral spastic CP. How disturbances in the sensory systems affect standing ability in children with bilateral CP will be further explored.

\section{Competing interests \\ The authors declare that they have no competing interests.}

\section{Authors' contribution}

$\mathrm{CL}$ participated in the design of the study, carried out the data collection, performed the statistical analyses, analyzed the data and drafted the manuscript. KT participated in the design of the study, analyzed the data, and actively improved and revised the manuscript. ÅB conceived of the study, carried out the data collection, analyzed the data, and actively improved and revised the manuscript. Each of the authors have read and concur with the content in the final manuscript.

Authors' information

Not applicable.

\section{Acknowledgements}

We would like to thank all the children and their parents who participated in this study which has been financially supported through grants from Stiftelsen Frimurare Barnahuset i Stockholm, Stiftelsen Promobilia, Sällskapet Barnavård and Linnéa och Josef Carlssons Stiftelse. 
Received: 9 February 2015 Accepted: 25 September 2015 Published online: 08 October 2015

\section{References}

1. Rosenbaum P, Paneth N, Leviton A, Goldstein M, Bax M, Damiano D, et al. A report: the definition and classification of cerebral palsy April 2006. Dev Med Child Neurol Suppl. 2007;109:8-14.

2. Eek MN, Beckung E. Walking ability is related to muscle strength in children with cerebral palsy. Gait Posture. 2008;28:366-71.

3. Wiley ME, Damiano DL. Lower-extremity strength profiles in spastic cerebral palsy. Dev Med Child Neurol. 1998;40:100-7.

4. Palisano R, Rosenbaum P, Walter S, Russell D, Wood E, Galuppi B. Development and reliability of a system to classify gross motor function in children with cerebral palsy. Dev Med Child Neurol. 1997;39:214-23.

5. Thompson N, Stebbins J, Seniorou M, Newham D. Muscle strength and walking ability in diplegic cerebral palsy: implications for assessment and management. Gait Posture. 2011;33:321-5.

6. Ross SA, Engsberg JR. Relationships between spasticity, strength, gait, and the GMFM-66 in persons with spastic diplegia cerebral palsy. Arch Phys Med Rehabil. 2007:88:1114-20.

7. Scholtes VA, Becher JG, Comuth A, Dekkers H, Van Dijk L, Dallmeijer AJ. Effectiveness of functional progressive resistance exercise strength training on muscle strength and mobility in children with cerebral palsy: a randomized controlled trial. Dev Med Child Neurol. 2010;52:e107-113.

8. Taylor NF, Dodd KJ, Baker RJ, Willoughby K, Thomason P, Graham HK. Progressive resistance training and mobility-related function in young people with cerebral palsy: a randomized controlled trial. Dev Med Child Neurol. 2013;55:806-12.

9. Ferrari A, Sghedoni A, Alboresi S, Pedroni E, Lombardi F. New definitions of 6 clinical signs of perceptual disorder in children with cerebral palsy: an observational study through reliability measures. Eur J Phys Rehabil Med. 2014;50:709-16.

10. Ferrari A, Tersi L, Sghedoni A, Chiari L. Functional reaching discloses perceptive impairment in diplegic children with cerebral palsy. Gait Posture. 2010;32:253-8.

11. Alboresi A, Belmonti V, Ferrari A, Ferrari A. Dysperceptive forms. In: Ferrari A, Cioni G, editors. The spastic forms of cerebral palsy : a guide to the assessment of adaptive functions. Milano: Springer; 2010. p. 273-90.

12. Damiano DL, Wingert JR, Stanley CJ, Curatalo L. Contribution of hip joint proprioception to static and dynamic balance in cerebral palsy: a case control study. J Neuroeng Rehabil. 2013;10:57.

13. Lidbeck CM, Gutierrez-Farewik EM, Brostrom E, Bartonek A. Postural orientation during standing in children with bilateral cerebral palsy. Pediatr Phys Ther. 2014;26:223-9.

14. Damiano DL, Dodd K, Taylor NF. Should we be testing and training muscle strength in cerebral palsy? Dev Med Child Neurol. 2002:44:68-72.

15. Verschuren O, Ketelaar M, Takken T, Van Brussel M, Helders PJ, Gorter JW. Reliability of hand-held dynamometry and functional strength tests for the lower extremity in children with Cerebral Palsy. Disabil Rehabil. 2008;30:1358-66.

16. Örtqvist M, Bartonek $\AA$, Gutierrez-Farewik E, Brostrom E. Knee muscle strength - A challenge to measure. Eur J Physiother. 2014;16:33-46.

17. Tedroff K, Knutson LM, Soderberg GL. Co-activity during maximum voluntary contraction: a study of four lower-extremity muscles in children with and without cerebral palsy. Dev Med Child Neurol. 2008;50:377-81.

18. Willemse L, Brehm MA, Scholtes VA, Jansen L, Woudenberg-Vos $H_{4}$ Dallmeijer AJ. Reliability of isometric lower-extremity muscle strength measurements in children with cerebral palsy: implications for measurement design. Phys Ther. 2013;93:935-41.

19. Crompton J, Galea MP, Phillips B. Hand-held dynamometry for muscle strength measurement in children with cerebral palsy. Dev Med Child Neurol. 2007;49:106-11.

20. American Academy of Orthopaedic Surgeons. Joint motion: method of measuring and recording. Edinburgh, London, Melbourne: Churchill Livingstone; 1988.

21. Bohannon RW, Smith MB. Interrater reliability of a modified Ashworth scale of muscle spasticity. Phys Ther. 1987;67:206-7.

22. Eek MN, Kroksmark AK, Beckung E. Isometric muscle torque in children 5 to 15 years of age: normative data. Arch Phys Med Rehabil. 2006;87:1091-9.

23. Tedroff K. Children with spastic cerebral palsy: aspects of muscle activity and botulinum toxin A treatment. Stockholm: Department of woman and child health, Karolinska institutet; 2009. p. 11-4.
24. Nordmark E, Hagglund G, Lauge-Pedersen H, Wagner P, Westbom L. Development of lower limb range of motion from early childhood to adolescence in cerebral palsy: a population-based study. BMC medicine. 2009;7:65.

25. Dallmeijer AJ, Baker R, Dodd KJ, Taylor NF. Association between isometric muscle strength and gait joint kinetics in adolescents and young adults with cerebral palsy. Gait Posture. 2011;33:326-32.

26. Wingert JR, Burton $H$, Sinclair RJ, Brunstrom JE, Damiano DL. Joint-position sense and kinesthesia in cerebral palsy. Arch Phys Med Rehabil. 2009;90:447-53.

27. Harms-Ringdahl K. Muscle strength. Edinburgh; New York: Churchill Livingstone; 1993.

28. Van Vulpen LF, De Groot S, Becher JG, De Wolf GS, Dallmeijer AJ. Feasibility and test-retest reliability of measuring lowerlimb strength in young children with cerebral palsy. Eur J Phys Rehabil Med. 2013;49:803-13.

\section{Submit your next manuscript to BioMed Central and take full advantage of:}

- Convenient online submission

- Thorough peer review

- No space constraints or color figure charges

- Immediate publication on acceptance

- Inclusion in PubMed, CAS, Scopus and Google Scholar

- Research which is freely available for redistribution 